\title{
Experimental Tests of Local Cosmological Expansion Rates
}

\author{
Allan Widom* and John Swain \\ Physics Department, Northeastern University \\ Boston, MA, 02115, USA \\ *E-mail: allan_widom@yahoo.com \\ john.swain@cern.ch \\ Yogendra Srivastava \\ Physics Department, University of Perugia \\ Perugia, Italy \\ E-mail: yogendra.srivastava@gmail.com
}

\begin{abstract}
Cosmological expansion on a local scale is usually neglected in part due to its smallness, and in part due to components of bound systems (especially those bound by nongravitational forces such as atoms and nuclei) not following geodesics in the cosmological metric. However, it is interesting to ask whether or not experimental tests of cosmological expansion on a local scale (well within our own galaxy) might be experimentally accessible in some way. We point out, using the Pioneer satellites as an example, that current satellite technology allows for this possibility within time scales less than one human lifetime.
\end{abstract}

Keywords: cosmology, local Hubble expansion, Pioneer

\section{Introduction}

The electromagnetic waves sent into our solar system by the Pioneer satellites 10 and 11 exhibit frequency shifts which vary with time $\underline{\underline{ }}$. Such frequency shifts were parameterized as accelerations dominated by the gravitational attraction between the solar system and the satellites. An anomalous acceleration remnant was reported which cannot be explained by the usual Newtonian gravitational field produced by the sun at the positions of the satellites.

To see what was involved, consider the velocity $\mathbf{v}(t)$ of a satellite moving away from the sun slowly into the milky way galaxy at a non-relativistic speed $v(t)=|\mathbf{v}(t)| \ll c$. What was expected was a Doppler shifted frequency of the electromagnetic wave which could be employed as an accelerometer;

$$
\omega(t)=\omega_{0}\left(1-\frac{v(t)}{c}+\cdots\right) \quad \Rightarrow \quad \frac{\dot{\omega}(t)}{\omega(t)}=-\frac{\dot{v}(t)}{c}+\cdots .
$$

The remnant experimental acceleration anomaly $-\mathcal{A}_{\infty}$ was reported $\underline{\underline{1}}$ as

$$
\mathcal{A}_{\infty}=\lim _{t \rightarrow \infty} \dot{v}(t) \approx 7.5 \times 10^{-8}\left[\frac{\mathrm{cm}}{\mathrm{sec}^{2}}\right],
$$

wherein $\infty$ denotes a time sufficiently large so that the gravitational acceleration towards the sun should have been virtually zero at least from the viewpoint of Newtonian gravitational theory. The experimental result reported in Eq.(2) was thereby thought by many to be an anomaly, if not an experimental error. For 
example, Turyshev et al. suggest a thermal origin ${ }^{2}$ of the data, but also acknowledge that open questions remain.

Regardless of whether or not one considers the Pioneer anomaly to be resolved (that is, explained by experimental factors now accounted for) or not, the important point we raise is that the level of precision is such that one can think about experimental tests of cosmological expansion on distance scales which can be reached by earth-launched satellites within one human lifetime.

\section{Uniform Cosmological Metrics}

The standard uniform cosmological metric may be written via the proper time

$$
c^{2} d \tau^{2}=c^{2} d t^{2}-a(t)^{2} d \ell^{2} \Rightarrow d \ell^{2}=d \chi^{2}+f_{\kappa}^{2}(\chi)\left[d \theta^{2}+\sin ^{2} \theta d \phi^{2}\right],
$$

wherein $\kappa=0, \pm 1, f_{+1}(\chi)=\sin \chi, f_{0}(\chi)=\chi$ and $f_{-1}(\chi)=\sinh \chi$. The Hubble expansion rate is thereby

$$
\mathcal{H}=\frac{\dot{a}}{a}
$$

A central issue in cosmology is to describe how the uniform metric in Eq. (3) can be employed to describe a very non-uniform distribution ${ }^{5}$ of observable gravitational masses. We follow convention in what follows, making no special claims about the possible non-uniformity or indeed presence of dark matter and dark energy, and simply take the standard cosmological metrics (which are applied with or without dark matter or energy) to be good approximations to parametrize cosmological metrics.

\section{Local Hubble Expansion}

According to general relativity and the standard cosmological models 3,4 , the universe is presently expanding at a Hubble rate $\mathcal{H}$ measured in part by the rate at which frequencies of electromagnetic radiation change with time

$$
\frac{\dot{\omega}}{\omega}=-\mathcal{H}=-\frac{1}{\mathcal{T}}
$$

where $\mathcal{T}$ is the Hubble time.

If a local Hubble expansion does exist, then the data should be parametrized by the assignment

$$
\frac{\mathcal{A}_{\infty}}{c}=\mathcal{H}=\frac{1}{\mathcal{T}} \quad \Rightarrow \quad \mathcal{T}=\frac{c}{\mathcal{A}_{\infty}}
$$

If one uses the Pioneer value (assuming it to be a real effect rather than an experimental artefact) of $\mathcal{A}_{\infty} \approx 7.5 \times 10^{-8}\left[\frac{\mathrm{cm}}{\mathrm{sec}^{2}}\right]$, together with the presence of Hubble expansion rate on a local length scale of the order of the Pioneer path length one obtains 


$$
\mathcal{T}=\frac{c}{\mathcal{A}_{\infty}} \approx 12.7 \times 10^{9} \text { year. }
$$

Rather remarkably, this is a numerical value for the Hubble time in good agreement with other measurements of this time which have been reviewed $\underline{4}$.

Thus, if one assumes the Pioneer acceleration to be a real effect, the gravitational acceleration toward the sun appears indeed virtually zero where and when it should be according to Newtonian gravity and the general relativistic Hubble expansion would appear to explain it.

Conversely, if the original apparent observed Pioneer acceleration is explained by experimental errors and actually zero, then this provides evidence against a local cosmological expansion.

Regardless of the interpretation of the data - and this is the key point of this paper - the precision which can be obtained from data from missions like the Pioneer ones appears sufficient to allow questions about local cosmological expansion to be experimentally tested, without having to wait for cosmological time scales, and indeed, well within the lifespan of a human being.

\section{Conclusion}

As noted in the abstract, cosmological expansion on a local scale is usually neglected in part due to its smallness, and in part due to components of bound systems not following geodesics in the cosmological metric. Nevertheless, as physics is an experimental subject, it is interesting to ask whether or not tests can be made within our cosmic environment using probes which we understand and can control.

We show, using the Pioneer satellites as an example, that current satellite technology allows for this possibility within time scales less than one human lifetime. Indeed the data, depending on whether or not the "anomaly" is considered to have been resolved, may already be good enough to start to address such questions.

More data and other experiments would be welcome (and indeed may be accessible through further analysis of Pioneer data and via the Deep Space Network ${ }^{2}$ ). At the very least, the results here indicate that tests of local Hubble expansion at relatively small cosmological distances are experimentally feasible.

\section{Acknowledgments}

J. S. would like to thank the United States National Science Foundation for support under PHY-1205845.

\section{References}

1. J. Anderson et. al., Phys. Rev. D 65, 082004 (2002).

2. S. G. Turyshev et al., Phys. Rev. Lett. 108, 241101 (2012). 
3. L.D. Landau and E.M. Lifshitz, "The Classical Theory of Fields", ButterworthHeinemann, Oxford (1999).

4. S. Weinberg, "Cosmology", Oxford University Press, Oxford (2008).

5. V. Springel, C.S. Frenk and S.D.M. White, Nature, 440, 1137 (2006). 